\title{
Gene silencing of TSPYL5 mediated by aberrant promoter methylation in gastric cancers
}

\author{
Yeonjoo Jung ${ }^{1}$, Jinah Park' ${ }^{1}$ Yung-Jue Bang ${ }^{1,2}$ and Tae-You Kim ${ }^{1,2}$
}

DNA methylation is crucial for normal development, but gene expression altered by DNA hypermethylation is often associated with human diseases, especially cancers. The gene TSPYL5, encoding testis-specific Y-like protein, was previously identified in microarray screens for genes induced by the inhibition of DNA methylation and histone deacetylation in glioma cell lines. The TSPYL5 showed a high frequency of DNA methylation-mediated silencing in both glioma cell lines and primary glial tumors. We now report that TSPYL5 is also inactivated by DNA methylation and could be a putative epigenetic target gene in gastric cancers. We found that the expression of TSPYL5 mRNA was frequently downregulated and inversely correlated with DNA methylation in seven out of nine gastric cancer cell lines. TSPYL5 mRNA expression was also restored after treating with a DNA methyltransferase inhibitor. In primary gastric tumors, methylation-specific PCR results in 23 of the 36 (63.9\%) cases revealed that the hypermethylation at CpG islands of the TSPYL5 was detectable at a high frequency. Furthermore, TSPYL5 suppressed the growth of gastric cancer cells as demonstrated by a colony formation assay. Thus, strong associations between TSPYL5 expression and hypermethylation were observed, and aberrant methylation at a CPG island of TSPYL5 may play an important role in development of gastric cancers.

Laboratory Investigation (2008) 88, 153-160; doi:10.1038/labinvest.3700706; published online 3 December 2007

KEYWORDS: DNMT inhibitor; gastric cancer; NAP domain; promoter hypermethylation; TSPYL5

Epigenetic events are heritable modifications that regulate gene expression and can contribute to cancer development. ${ }^{1}$ In particular, changes in DNA methylation are among the most common molecular alterations in human neoplasia, because the hypermethylation of a tumor suppressor gene at a promoter can lead to transcriptional silencing and the loss of gene function. ${ }^{1-4}$ Gastric cancer is one of the most common malignancies in the world. ${ }^{5}$ The promoter hypermethylation of multiple tumor suppressor genes resulting in gene silencing has been recognized as an important mechanism in gastrointestinal carcinogenesis. ${ }^{6-15}$

In a previous study, TSPYL5 was identified as one of the genes induced after treatment with DNA methylation and histone deacetylation inhibitors in glioma cell lines using microarray analysis. ${ }^{16}$ The TSPYL5 showed a high frequency of DNA methylation-mediated silencing in both glioma cell lines and primary glial tumors. A human TSPYL5 encoding testis-specific Y-like protein 5 (TSPYL5) is located on chromosome 8q22.1, but its role in human cancer has not been fully understood. The TSPYL 5 protein is a member of TSPYL family that includes TSPYL1, TSPYL2, TSPYL3, TSPYL4, and
TSPYL6. TSPYL proteins are also members of the nucleosome assembly protein (NAP) superfamily. The TSPYLs show sequence homology to NAPs, which have a highly conserved domain (the NAP domain) of approximately 180 amino acids. ${ }^{17,18}$ The NAP domain is known to be required and sufficient for histone binding. ${ }^{19}$ Members of the NAP family of proteins were identified in different systems and were classified with the NAPs based on their sequence homology and the possession of the NAP domain rather than their functions. The expression of NAP proteins is highly specific for many cell types and tissues. ${ }^{16-18,20,21}$ In humans, the NAP family members include NAP1, NAP1-like protein (NAP1L), SE translocation (SET), testis-specific protein, Y-encoded (TSPY), and testis-specific protein, Y-encoded like (TSPYL). ${ }^{17,20,22-26}$ It is known that proteins in the NAP family participate in chromatin structure and diverse cellular events, and may be involved in regulating gene expression as a result of histone accessibility. ${ }^{17}$ NAP1, which is the best characterized member of the NAP family, plays a role in the exchange process of histone $\mathrm{H} 2 \mathrm{~A}$ and $\mathrm{H} 2 \mathrm{~B}$ dimers from assembled nucleosomes in DNA replication as a histone

\footnotetext{
Laboratory for Cancer Epigenetics, Department of Tumor Biology, Cancer Research Institute, Seoul National University College of Medicine, Seoul, Korea and

${ }^{2}$ Department of Internal Medicine, Seoul National University College of Medicine, Seoul, Korea

Correspondence: Dr T-Y Kim, MD, PhD, Department of Internal Medicine, Seoul National University College of Medicine, 28 Yongon-dong, Chongno-gu, Seoul 110-744, South Korea. E-mail: kimty@snu.ac.kr

Received 04 July 2007; revised 25 October 2007; accepted 26 October 2007
} 
chaperone, and NAP1 may assist nucleosome sliding. ${ }^{24}$ NAP1 is also known to be involved in nucleocytoplasmic shuttling in mitotic progression in yeast. ${ }^{27}$ TSPY functions in early spermatogenesis and testicular tumorigenesis, ${ }^{21}$ and potentiates cell proliferation and tumorigenesis by promoting cell-cycle progression in some cancer cells. ${ }^{28}$ TSPYL2 (also known as CDA1, CINAP, or DENTT) facilitates chromatin assembly by binding to core histones in vitro and arrests cell growth. ${ }^{29-31}$ Only a few studies have reported inactivation of the NAP-related protein in vitro. The downregulation of the TSPY gene in melanoma progression is caused by promoter hypermethylation. ${ }^{32}$ A recent study showed that sudden infant death with dysgenesis of the testes syndrome (SIDDT, OMIM no. \#608800) was due to a frameshift mutation in the TSPYL1 gene, and this resulted in truncation at codon 169 leading to the loss of a region with high homology to the NAP domain. ${ }^{18}$

The purpose of this study was to investigate gene silencing of the TSPYL5 mediated by promoter hypermethylation in gastric cancers, and the correlation between the inactivation of TSPYL5 and gastric cancers. We evaluated the methylation status of a CpG island in the promoter and first exon of TSPYL5 and also used a demethylating agent to reactivate gene expression. Our data indicate that the TSPYL5 is frequently inactivated by hypermethylation and could be a candidate gene for epigenetic targeting in gastric cancers.

\section{MATERIALS AND METHODS}

\section{Cell Culture and Tissue Samples}

Human gastric adenocarcinoma SNU-1, SNU-16, SNU-484, SNU-601, SNU-620, SNU-638, SNU-668, and SNU-719 cell lines were obtained from the Korean cell line bank. ${ }^{33}$ AGS and MDA-MB-231 cells were obtained from the American Type Culture Collection (Manassas, VA, USA). All cell lines were cultured in RPMI-1640 supplemented with $10 \%$ fetal bovine serum (WelGene, Korea). Gastric carcinoma tumor tissue samples and matching non-cancerous tissues were obtained from the Seoul National University Hospital (Seoul, Korea), and the frozen tissue samples were handled as described earlier. ${ }^{15}$

Treatment of 5-Aza-2'-deoxycytidine and Trichostatin A One day after seeding, cells were treated with 5-aza-2'deoxycytidine (5-aza-dC; Sigma, St Louis, MO, USA) at $5 \mu \mathrm{M}$ or at different concentrations $(0.1,0.5$ and $1 \mu \mathrm{M})$ for 4 days. The medium including 5-aza-dC was replaced every other day. Cells were exposed on trichostatin A (TSA; Wako, Japan) at $0.1 \mu \mathrm{M}$ for $24 \mathrm{~h}$. For cotreatment with 5 -aza-dC and TSA, cells were exposed on both agents for an additional $24 \mathrm{~h}$ after a single treatment with 5 -aza-dC for $96 \mathrm{~h}$.

\section{RT-PCR Analysis}

Total RNA was isolated using TRI Reagent ${ }^{\mathbb{R}}$ (Molecular Research Center, Cincinnati, OH, USA) according to the manufacturer's instructions. cDNA was synthesized from $2 \mu \mathrm{g}$
Table 1 Primer sequences for RT-PCR analysis

\begin{tabular}{|c|c|c|c|c|}
\hline Gene & & Primer sequence $\left(5^{\prime} \rightarrow 3^{\prime}\right)$ & NCBI no. & Length (bp) \\
\hline \multirow[t]{2}{*}{ TSPYL1 } & $\mathrm{F}$ & AGTGGCGGAGGAGGATAGAT & NM_003309 & 274 \\
\hline & $\mathrm{R}$ & CCTAATCATGGCGGACAACT & & \\
\hline \multirow[t]{2}{*}{ TSPYL2 } & $\mathrm{F}$ & CAATGAGGGCAGTGATGATG & NM_022117 & 262 \\
\hline & $\mathrm{R}$ & АTCTTCСССТTСTTCCCAGA & & \\
\hline \multirow[t]{2}{*}{ TSPYL3 } & $\mathrm{F}$ & TCAGCTGGTITCACAGCAC & BC101558 & 173 \\
\hline & $\mathrm{R}$ & GAACCCGTAGGGCCTAGAAG & & \\
\hline \multirow[t]{2}{*}{ TSPYL4 } & $\mathrm{F}$ & CTACGGCTTCTATGCCAAGC & NM_021648 & 281 \\
\hline & $\mathrm{R}$ & GGAATGTCCAATTGGGTTG & & \\
\hline \multirow[t]{2}{*}{ TSPYL5 } & $\mathrm{F}$ & ATCCCCAGCTAGCATCCTIT & NM_033512 & 219 \\
\hline & $\mathrm{R}$ & GGAGCCACTGGATTGGAGTA & & \\
\hline \multirow[t]{2}{*}{ TSPYL6 } & $\mathrm{F}$ & GCTITCCTGTCAGCACACAA & NM_001003937 & 235 \\
\hline & $\mathrm{R}$ & GGAAGGGAGGCAAATAAAGG & & \\
\hline \multirow[t]{2}{*}{$\beta$-Actin } & $\mathrm{F}$ & TCATCACCATTGGCAATGAG & BC013835 & 155 \\
\hline & $\mathrm{R}$ & CACTGTGTTGGCGTACAGGT & & \\
\hline
\end{tabular}

F, forward primers; $R$, reverse primers; all primer sequences were designed by using Primer3 program.

of total RNA using ImProm-II ${ }^{\mathrm{TM}}$ reverse transcriptase (Promega, Madison, WI, USA). The cDNA was amplified by using rTaq DNA polymerase (Takara, Japan) under the following conditions: $94^{\circ} \mathrm{C}$ for $5 \mathrm{~min}, 29-32$ cycles of $94^{\circ} \mathrm{C}$ for $30 \mathrm{~s}, 60^{\circ} \mathrm{C}$ for $30 \mathrm{~s}$, and $72^{\circ} \mathrm{C}$ for $30 \mathrm{~s}$, and the final extension of $72^{\circ} \mathrm{C}$ for $10 \mathrm{~min}$. Amplification of $\beta$-actin from cDNA samples was used as an internal control for the reverse transcription-PCR (RT-PCR) reaction. The primer sequences are listed in Table 1 . The amplified PCR products were separated on $2 \%$ agarose gels.

\section{Methylation-Specific PCR and Bisulfite Sequencing}

We isolated genomic DNA from cells was isolated using the DNA extraction kit (Intron Biotechnology, Korea). Genomic DNA from frozen tumor samples and paired normal tissue samples was isolated by standard phenol-chloroform extraction and ethanol precipitation methods. Based on the previous methods, ${ }^{34} 1 \mu \mathrm{g}$ of genomic DNA was treated with sodium bisulfite by using a kit from In2Gen (Korea). To determine the methylation status of the promoter region of TSPYL5, a CpG island region was searched and the specific primers for methylation-specific PCR (MSP) were designed by using MethPrimer program. ${ }^{35}$ Primer sequences for MSP of the TSPYL5 are as follows: 5'-GAGCGTATATTAGA GAAATTCGTCGA- $3^{\prime}$ (F) and 5'-GCTATAACCCTACGACTC CTAACG- $3^{\prime}(\mathrm{R})$ for methylated DNA, and $5^{\prime}$-GAGTGTATAT TAGAGAAATTTGTTGA-3' $3^{\prime}$ (F) and $5^{\prime}$-ACTATAACCCTA CAACTCCTAACACC-3' (R) for unmethylated DNA. PCR amplification was performed with AmpliTaq ${ }^{\mathbb{R}}$ Gold DNA polymerase (Applied Biosystems, Foster, CA, USA) under the following conditions: $95^{\circ} \mathrm{C}$ for $10 \mathrm{~min}, 30-33$ cycles of $94^{\circ} \mathrm{C}$ 
for $30 \mathrm{~s}, 60^{\circ} \mathrm{C}$ for $30 \mathrm{~s}$, and $72^{\circ} \mathrm{C}$ for $30 \mathrm{~s}$, and the final extension of $72^{\circ} \mathrm{C}$ for $10 \mathrm{~min}$. For a statistical analysis of the MSP results of primary gastric tissues, Fisher's exact test, the $t$-test and $\chi^{2}$-test were used to determine the significance of associations between different variables by using SPSS $12.0 \mathrm{~K}$ software. The level of statistical significance was $P<0.05$. For bisulfite sequencing analysis, the promoter region of TSPYL5 (from -182 to +94 ) was sequenced after PCR amplification with specific primers, $5^{\prime}$-GAAGAGATGAAATGGTAGTAT- ${ }^{\prime}$ (F) and $5^{\prime}$-AAAACAACTTCAAAAACAC- $3^{\prime}$ (R) using an AmpliTaq ${ }^{\circledR}$ Gold DNA polymerase. The amplified PCR products were subcloned into $\mathrm{pCR}^{\circledR} 2.1$ TOPO (Invitrogen, Carlsbad, CA, USA) and the DNA insert was sequenced using an ABI PRISM 3730 DNA analyzer (Applied Biosystems).

\section{Construction of Expression Plasmids}

pcDNA3-TSPYL5 was constructed by subcloning the PCR product generated from the full-length clone for the TSPYL5 sequence (NCBI no. BC045630) obtained from Open Biosystems (Huntsville, AL, USA) with Takara Ex Taq DNA polymerase. PCR primers were designed to produce the full length of a coding region of the TSPYL5 protein, with recognition sites of the restriction enzymes (EcoRI and XbaI) at the ends. The sequence of construct and proper orientations were confirmed by sequencing. An expression plasmid for the CDKN2A gene has been used as described previously. ${ }^{16}$

\section{Transfection and Colony Formation}

For colony formation assay using monolayer culture, cells were seeded at a density of $2.5 \times 10^{5}$ cells per well in six-well plates. Cells were transfected with TSPYL5 expression plasmids or the empty vector ( $2 \mu \mathrm{g}$ each) using Lipofectamine 2000 (Invitrogen) according to the manufacturer's instructions. Forty-eight hours after transfection, the transfectants were selected using G418 (Cellgro, Herndon, VA, USA) at $600 \mu \mathrm{g} / \mathrm{ml}$. After 10-14 days, the colonies were washed with PBS, fixed with absolute methanol, and stained with a crystal violet solution $(0.5 \%)$ for $20 \mathrm{~min}$. At least 150 of surviving colonies for the control (empty vector) were counted using a Bio-Rad gel documentation system. Total RNA from the transfected cells was isolated and analyzed the overexpression of TSPYL5 by RT-PCR. All assays were repeated three times, and data are presented as the mean \pm s.d.

\section{RESULTS}

\section{The Loss of TSPYL5 Expression and Restoration by a Demethylating Agent in Gastric Cancer Cells}

The mRNA expression of the TSPYL genes was analyzed in human gastric cancer cell lines by RT-PCR analysis (Figure 1a). Unlike other members of the TSPYL family (TSPYL1, TSPYL2, TSPYL3, TSPYL4, and TSPYL6), the mRNA expression of the TSPYL5 reflected gene silencing in some cancer cell lines. Among the nine gastric carcinoma cell lines, two cell lines (SNU-484 and SNU-668) expressed TSPYL5
mRNA, while seven cell lines (SNU-1, SNU-601, SNU-620, SNU-638, SNU-719, AGS, and MKN28) did not express TSPYL5 mRNA. We also examined the mRNA expression of the TSPYLs in normal gastric tissue and observed that all of the TSPYL mRNAs were expressed (Figure 1a). To investigate whether demethylation could restore the loss of the TSPYL5 expression in nonexpressing cells, we used the demethylating agent 5-aza-dC. Seven TSPYL5-nonexpressing cells (SNU-1, SNU-601, SNU-620, SNU-638, SNU-719, AGS, and MKN28) were treated with $5 \mu \mathrm{M}$ of 5 -aza-dC for 4 days, and the mRNA expression levels of TSPYL5 were analyzed (Figure $1 \mathrm{~b})$. The addition of 5-aza-dC resulted in the restoration of TSPYL5 mRNA in all seven TSPYL5-nonexpressing cells. Moreover, TSPYL5 mRNA was induced dose dependently in SNU-601 cells after exposure to increasing concentrations of 5 -aza-dC for 4 days (Figure 1c). These results suggest that the loss of the TSPYL5 expression is caused by CpG methylation of the promoter sites and that the expression of the TSPYL5 is activated by the demethylating agent. To confirm whether the activities of both DNA methyltransferase and histone deacetylase play a role in the loss of TSPYL5 expression, two epigenetic inhibitors were used in combination. SNU-719 cells were exposed on 5-aza-dC at $5 \mu \mathrm{M}$ and TSA at $0.1 \mu \mathrm{M}$ to for 4 days and an additional $12 \mathrm{~h}$. We observed that the combined treatment showed additive induction of TSPYL5 mRNA compared with cells treated with 5-aza-dC alone. The treatment with TSA alone for $24 \mathrm{~h}$ showed no effect on re-expression of TSPYL5 mRNA (Figure 1d).

\section{Promoter Methylation of TSPYL5 in Gastric Cancer Cells} $\mathrm{CpG}$ promoter methylation is one of the causes of the gene silencing of tumor suppressor genes. For methylation analysis, the sequence from $1 \mathrm{~kb}$ upstream of the translation start site to first exon of TSPYL5 was analyzed using the MethPrimer program. ${ }^{35}$ The criteria for a CpG island were a high GC percentage ( $>60 \%$ ) and more over 0.7 of observed/ expected $\mathrm{CpG}$ ratio. As a result, the 925-bp fragment around the transcriptional start site (TSS) of the TSPYL5 was revealed as a $\mathrm{CpG}$ island satisfying the criteria (Figure 2a). To determine the potential for CpG methylation of TSPYL5, the methylation status was analyzed by MSP in the gastric cancer cell lines. As shown in Figure 2b, no band for CpG methylation was observed in SNU-484 and SNU-668 cells that congenitally express TSPYL5 mRNA, whereas the TSPYL5nonexpressing cell lines (SNU-1, SNU-601, SNU-620, SNU638, SNU-719, AGS, and MKN28) showed a pattern of complete methylation. To test whether the restoration of TSPYL5 mRNA expression is associated with a change in the methylation status of TSPYL5, MSP analysis was carried out on genomic DNA of 5-aza-dC-treated cells (Figure 2c). SNU-1 cells treated with 5-aza-dC showed the appearance of an unmethylated band, which was absent in untreated cells, and a similar result was seen in AGS cells. We believe that this result can be attributed to the demethylation effect at a $\mathrm{CpG}$ island of TSPYL5. For a delicate methylation analysis, we 
a

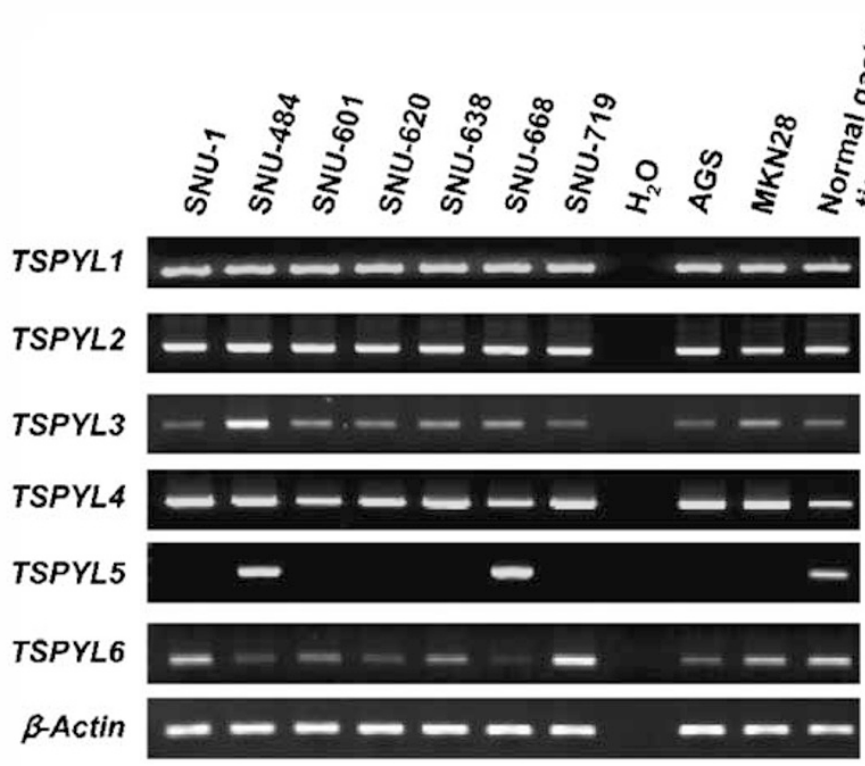

C
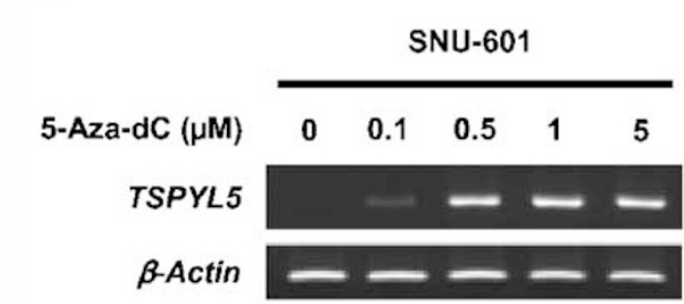

d

5-Aza-dC $(5 \mu \mathrm{M})$

TSA (100 nM)

TSPYL5

B-Actin

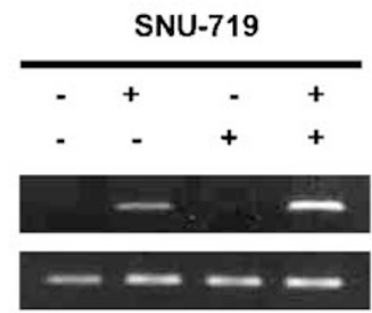

b

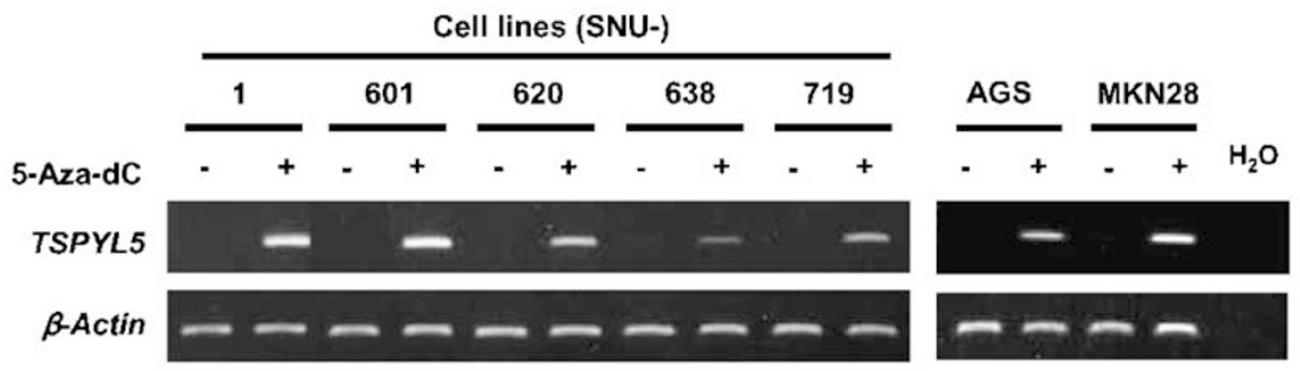

Figure 1 The mRNA expression of the TSPYL genes, and the epigenetic loss of TSPYL5. (a) The mRNA expression of the TSPYLs. Gene silencing is apparent only in the TSPYL5 mRNA. $\beta$-Actin was used as a loading control for RT-PCR. (b) RT-PCR analysis of TSPYL5 after treating with 5-aza-dC for 4 days. (c) SNU-601 cells were exposed to $0.1,0.5,1$, or $5 \mu \mathrm{M}$ of 5 -aza-dC for 4 days. (d) The combinatorial treatment of SNU-719 cells with 5 -aza-dC at $5 \mu \mathrm{M}$ and TSA at $0.1 \mu \mathrm{M}$.
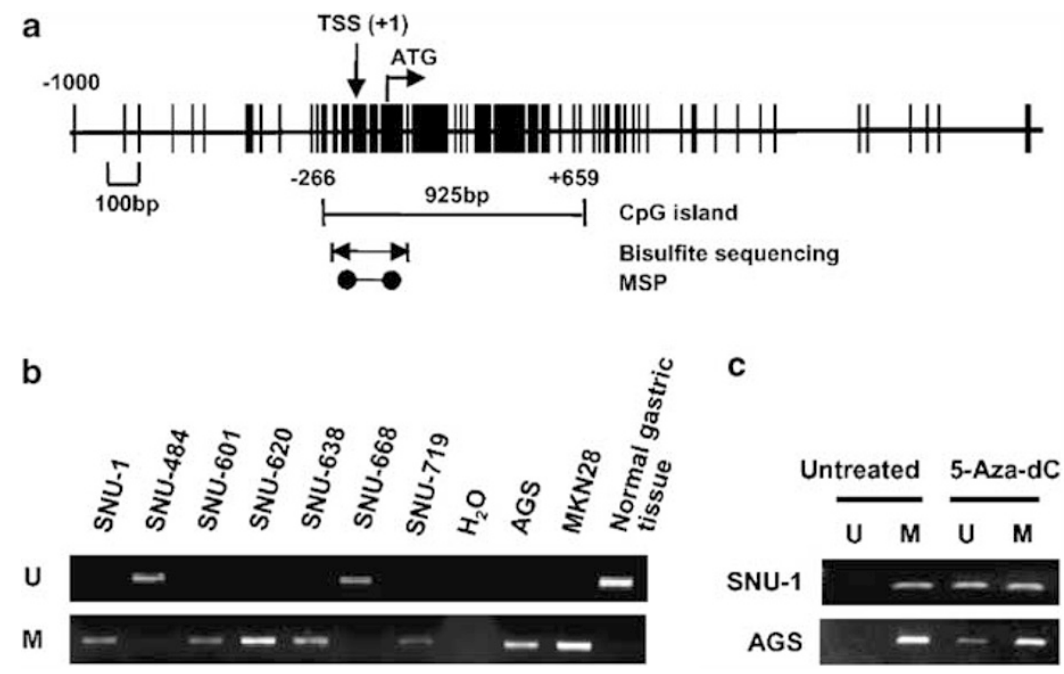

Figure 2 Methylation status at a CpG island of the TSPYL5 in gastric cancer cell lines. (a) Schematic structure of the TSPYL5 and location of a CpG island. Vertical bars represent the locations of CpG sites and locations investigated by MSP and bisulfite sequencing in this study are indicated. TSS means transcriptional start site and ATG indicates the start point for translation. (b) MSP analysis from bisulfite-modified DNA in gastric cancer cells with primers specific for unmethylated (U) or methylated (M) DNA. (c) Demethylation was analyzed by MSP in SNU-1 and AGS cells before and after the exposure to 5 -aza-dC at $5 \mu \mathrm{M}$ for 4 days. 
SNU-601
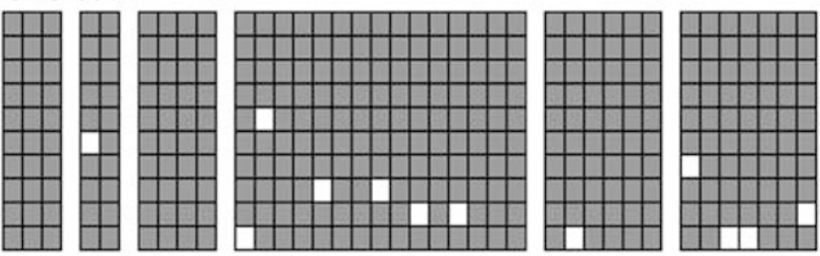

SNU-668
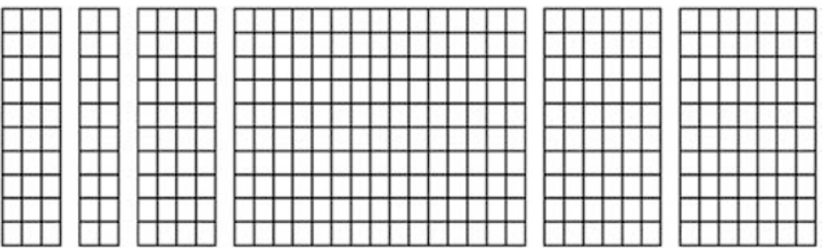

SNU-1
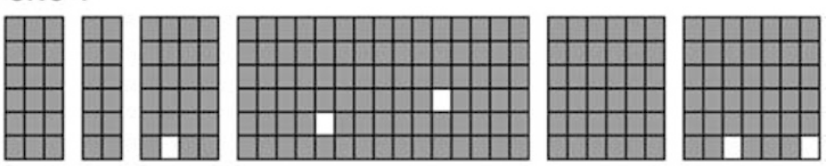

SNU-484
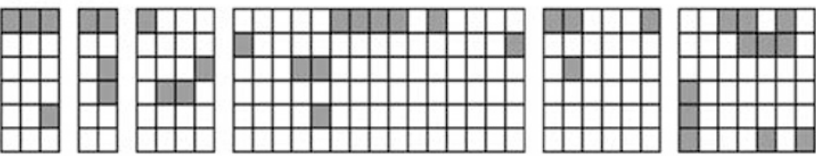

AGS
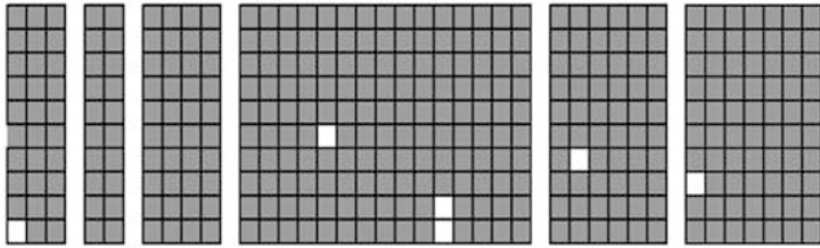

Normal gastric tissue
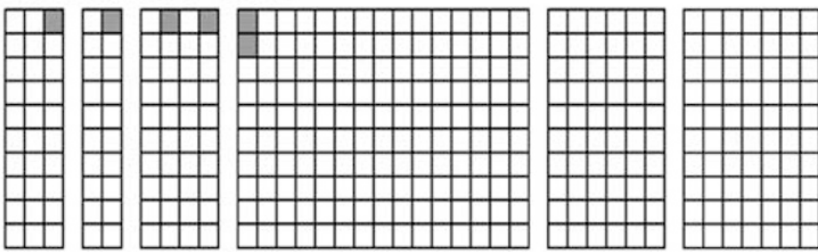

Figure 3 Bisulfite sequencing results at a CpG island of TSPYL5 in gastric cancer cell lines. The methylation status of the $37 \mathrm{CpG}$ sites at the CpG island of TSPYL5 was analyzed by sequencing. Each square indicates a $\mathrm{CpG}$ site, and each line of squares represents a single cloned allele. Open and closed squares represent unmethylated and methylated CpG sites, respectively.

used bisulfite genomic sequencing about the 276-bp amplified PCR product of the bisulfite-modified genomic DNA containing $37 \mathrm{CpG}$ sites on the promoter at the TSPYL5 (Figure 3). SNU-484 and SNU-668 cells showed a low frequency of methylation at the amplified region of the TSPYL5 (17\% in SNU-484 and 0\% in SNU-668 cells). However, in consistence with the MSP results (Figure 2b), CpG islands in SNU-1, SNU-601 and AGS cells were nearly completely
Table 2 Clinicopathological features of $\mathbf{3 6}$ gastric cancer patients and methylation status of TSPYL5 in cancer tissues and matching non-cancerous tissues

Case no. Methylation Unmethylation P-value

(\%)

(\%)

\begin{tabular}{lrrr}
\hline Non-cancer & 36 & $5(14)$ & $31(86)$ \\
Cancer & 36 & $23(64)$ & $13(36)$
\end{tabular}

Gender

Male

24

$16(67)$

8 (33)

Female

12

7 (58)

5 (42)

Age (mean, years)

$57.4 \pm 11.8$

$51.2 \pm 12.0$

$0.14^{\mathrm{b}}$

Differentiation

$$
\text { Poor }
$$

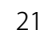

$13(62)$

8 (38)

Moderate/well

15

$10(67)$

5 (33)

Tumor stage

$$
\text { T1 }
$$

$\mathrm{T} 2$

T3

T4

Lymph nodes

\section{Negative}

Positive

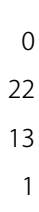

0
$16(44.4)$
$6(16.7)$
$1(2.8)$

0

$6(16.7)$

7 (19.4)

0

$0.27^{\mathrm{a}}$

$\begin{array}{rr}8(80) & 2(20) \\ 15(58) & 11(42)\end{array}$

${ }^{\mathrm{a}}$ Fisher's exact test; ${ }^{\mathrm{b}}$ t-test; ${ }^{\mathrm{c}} \chi^{2}$-test.

methylated (98, 97, and 98\%, respectively) at the amplified region of the TSPYL5. Taken together, these results indicate that the methylation status at a CpG island of the TSPYL5 is closely correlated with its transcriptional silencing in gastric cancer cells.

\section{Aberrant Methylation of TSPYL5 in Primary Gastric Cancer Tissues}

We next examined whether a CpG island of the TSPYL5 is also methylated in primary gastric cancer tissues as seen in vitro. Table 2 shows the results of the MSP analysis for the specific region of the TSPYL5 obtained from 36 primary tumor samples. The results indicate that the $\mathrm{CpG}$ island of TSPYL5 was hypermethylated in 23 cases of 36 primary gastric cancer tissues (64\%). Figure $4 \mathrm{a}$ shows a representative subset of the MSP results from the 36 primary tumor tissues and corresponding non-cancerous gastric tissues. The frequency of hypermethylation in non-cancerous gastric tissues was significantly lower than that in primary gastric cancer tissues regarding the analyzed region. Since most cancer tissues showed the coexistence of both unmethylation and methylation, we could not rule out a possibility of contamination of non-cancerous cells in gastric cancer tissue, which appeared to be methylated and also unmethylated. By bisulfite sequencing analysis, we examined the methylation 
a
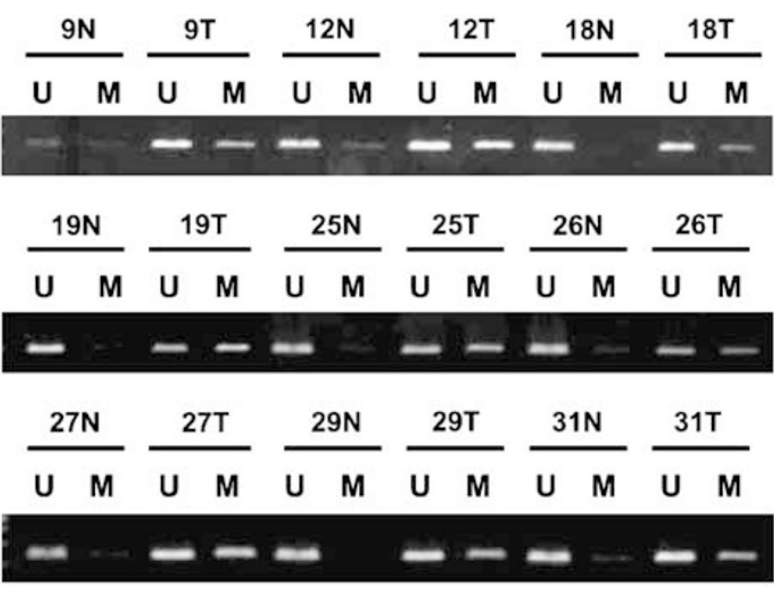

b

$19 N$
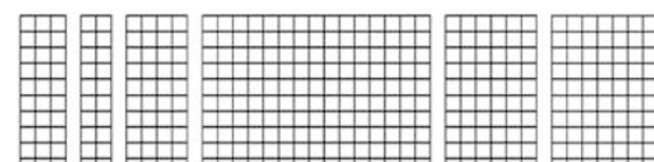

$19 T$
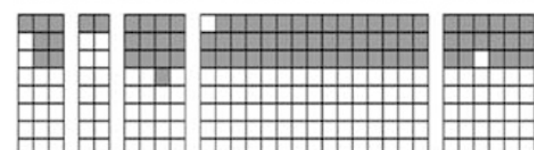

$25 \mathrm{~N}$
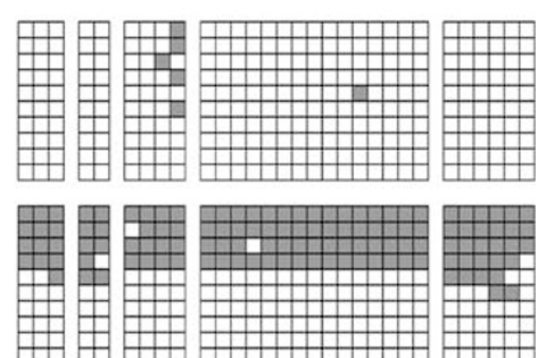

Figure 4 Methylation analysis at a CpG island of TSPYL5 in primary gastric cancers. (a) The representative MSP results at a CpG island of TSPYL5 in primary gastric tissues. Parallel amplification reactions in cancer tissue samples and matching non-cancerous tissue samples were performed using primers specific for unmethylated (U) or methylated (M) DNA. (b) The methylation status of TSPYL5 was determined by bisulfite sequencing in cancer tissue samples and corresponding non-cancerous tissue samples. Each line of squares represents a single cloned allele. T, tumor tissue DNA; $\mathrm{N}$, non-cancerous tissue DNA.

status of the 276-bp bisulfite-modified genomic DNA region containing $37 \mathrm{CpG}$ sites of the TSPYL5 from gastric cancer tissues and paired non-cancerous tissues (Figure 4b). The bisulfite sequencing results of two representative pairs from primary gastric tissues show that the methylation level was greatly increased in cancer regions (three of eight and four of 10) than corresponding non-cancer regions (zero of 10 and zero of 10 , respectively). The findings demonstrate that methylation at TSPYL5 promoter can be observed as a relatively common event in gastric cancers as seen in vitro. The clinical and pathological data in gastric cancer tissues and matching non-cancerous tissues are summarized in Table 2. Statistical analysis of the MSP results was performed to determine the significance of associations between different variables. However, methylation at TSPYL5 promoter in primary gastric cancer tissue was not significantly correlated with gender, age, differentiation, tumor stage or lymph node stage of patients.

\section{Growth Suppression by the Restoration of TSPYL5}

Based on the correlation between the loss of expression and DNA methylation of the TSPYL5 in gastric cancers, we examined whether the expression of TSPYL5 affects a growth-suppressive property in human gastric cancer cells. The effect of TSPYL5 expression on cell growth was assessed by using a colony formation assay (Figure 5 ). We cloned the full-length cDNA of TSPYL5 into the pcDNA3 vectors, and the expression plasmids were transfected into the SNU-601 and SNU-719 cell lines. G418 was added for 2 weeks to select only the transfectants. The number of colonies of transfectants with empty vector as a negative control was considered to be $100 \%$. The transfection of CDKN2A was used as a positive control and resulted in the significant suppression of growth in cancer cells. The expression of TSPYL5 after transfection was confirmed by real-time RT-PCR (data not shown). The growth inhibitory effect of TSPYL5 was further confirmed by stable transfection experiments and soft agar assay (Supplementary data). The results show that the overexpression of TSPYL5 yields a significant growth-suppressive effect $(39.8 \%$ in SNU-601 and $62.4 \%$ in SNU-719 cells), suggesting that the TSPYL5 may have the properties of a tumor suppressor gene in gastric cancers.

\section{DISCUSSION}

Silencing of tumor suppressor genes by aberrant methylation at $\mathrm{CpG}$ islands plays a crucial role in the development of various cancers. Many genes that are involved in the regulation of cell cycle, tissue invasion, DNA repair, and apoptosis have been shown to be inactivated by promoter hypermethylation in gastric cancer. ${ }^{6,7}$

In this study, we have shown that the mRNA expression of the TSPYL5 was significantly reduced or absent in seven of nine gastric cancer cell lines compared with normal gastric tissue (Figure 1a). We found that the TSPYL5 promoter was methylated in gastric cancer cells as demonstrated by methylation-detectable MSP analysis (Figure 2b). The assessment of DNA methylation by bisulfite sequencing also convincingly demonstrated that the promoter site on genomic DNA of TSPYL5 was methylated with a high frequency in gastric cancer cells (Figure 3). These results also showed that the DNA methylation status of TSPYL5 was inversely matched with the loss of expression. We found that the use of a demethylating agent (5-aza-dC) for 4 days could restore TSPYL5 expression (Figure 1b). Furthermore, the CpG promoter of TSPYL5 is methylated in the majority of gastric cancer cell lines $(77.8 \%)$, and also in that of primary gastric tumors (63.9\%) (Figure 2b; Table 2). These results 

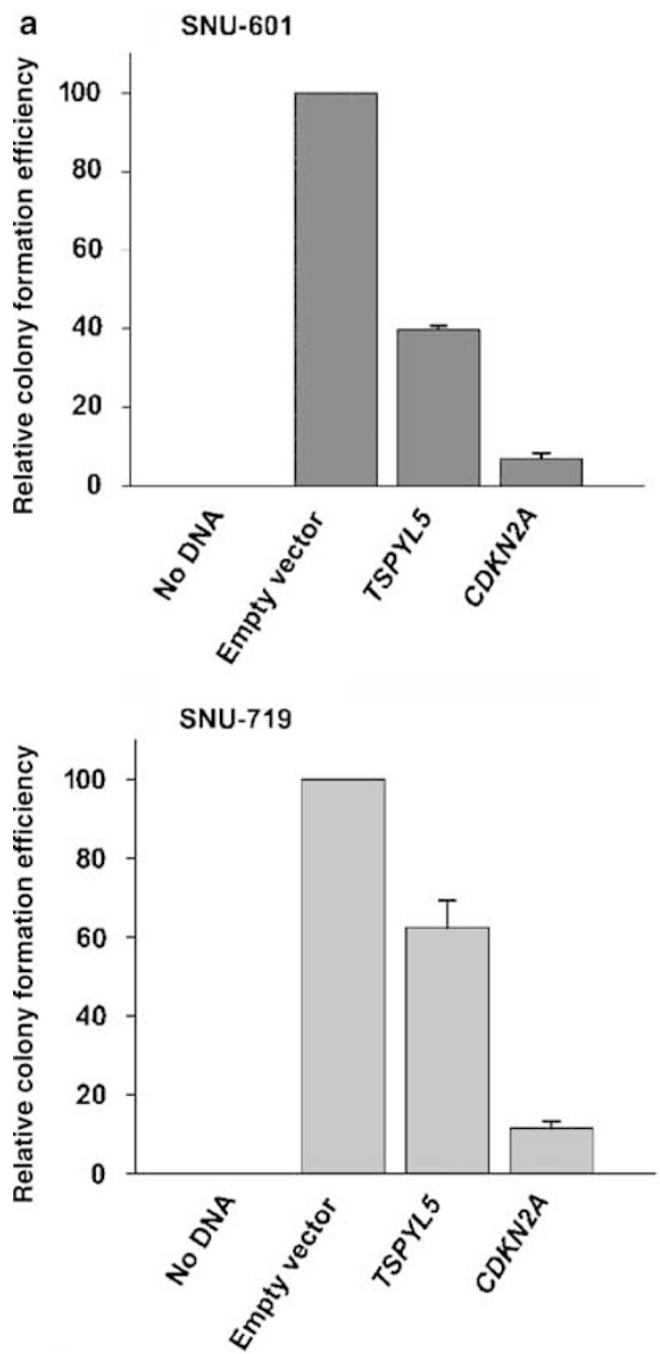

SNU-601

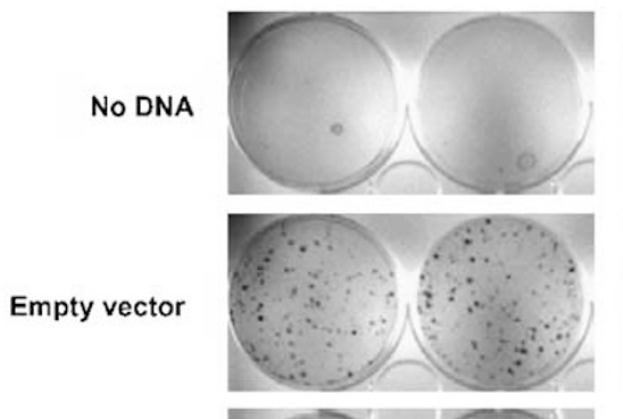

TSPYL5

CDKN2A
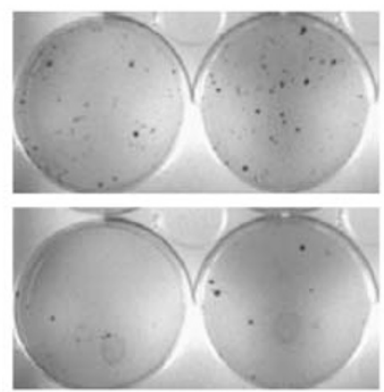

SNU-719
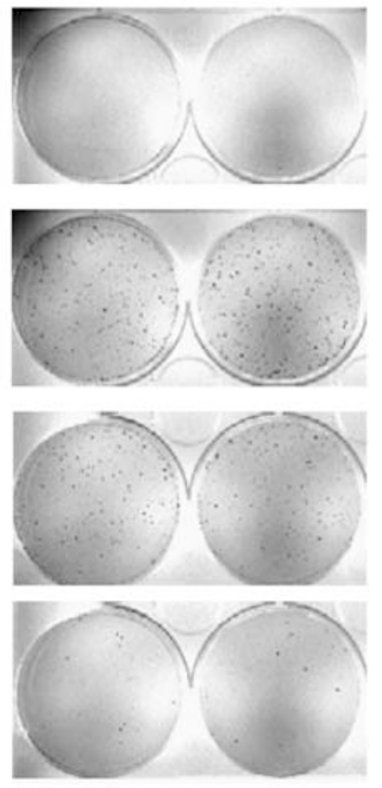

Figure 5 Growth-suppressive activity of TSPYL5. (a) SNU-601 and SNU-719 cells were transfected with $2 \mu \mathrm{g}$ of a TSPYL5 or a CDKN2A (a positive control) expression plasmids or an empty vector. Colonies were stained and counted 14-20 days after G418-addition at $600 \mu \mathrm{g} / \mathrm{ml}$. Values are means $\pm \mathrm{s}$.d. of triplicate plates. In three separate experiments, a similar result was observed. (b) The representative results of colony formation assays in SNU-601 and SNU719 cells.

demonstrate that DNA methylation at a $\mathrm{CpG}$ island of the TSPYL5 may be involved in the mechanism of gene silencing.

We examined the cooperative effects of DNA methylation and histone deacetylation by using two inhibitors (5-aza-dC and TSA) on SNU-719 cells. We found that cotreatment with the two agents synergistically increased the level of TSPYL5 mRNA compared with 5-aza-dC alone (Figure 1d). This result supports that DNMT and HDAC cooperatively could play an essential role in transcriptional silencing of several methylated genes. ${ }^{36}$ However, the recovery of histone acetylation using TSA alone did not induce the mRNA expression of TSPYL5. This result suggests that DNA methylation might play a dominant role over histone deacetylation in the repression of TSPYL5 and that histone deacetylation might have a modulating effect.

In summary, our findings demonstrated that $\mathrm{CpG}$ sites of the TSPYL5 are frequently methylated in gastric cancers and that hypermethylation at the promoter is significantly associated with silencing of TSPYL5. Although further evidence is needed to fully understand the molecular mechanism of TSPYL5 as a tumor suppressor gene, our data show that the expression of TSPYL5 suppresses the growth of gastric cancer cells, suggesting that it is a tumor suppressor candidate. Our results indicate that epigenetic mechanisms mediate the loss of TSPYL5 expression and that DNA methylation may play an important role in human gastric carcinogenesis.

Supplementary Information accompanies the paper on the Laboratory Investigation website (http://www.laboratoryinvestigation.org)

\section{ACKNOWLEDGEMENT}

This work was supported in part by grants from the Korean Ministry of Science and Technology through the National Research Laboratory Program for Cancer Epigenetics (no. M10400000336-06J0000-33610), and BK21 Project for Medicine, Dentistry and Pharmacy. 
1. Baylin SB. DNA methylation and gene silencing in cancer. Nat Clin Pract Oncol 2005;2(Suppl 1):S4-S11.

2. Jones PA, Baylin SB. The fundamental role of epigenetic events in cancer. Nat Rev Genet 2002;3:415-428.

3. Rountree MR, Bachman KE, Herman JG, et al. DNA methylation, chromatin inheritance, and cancer. Oncogene 2001;20:3156-3165.

4. Issa JP. CpG island methylator phenotype in cancer. Nat Rev Cancer 2004;4:988-993.

5. Hohenberger P, Gretschel S. Gastric cancer. Lancet 2003;362:305-315.

6. Kang GH, Lee $\mathrm{S}$, Kim JS, et al. Profile of aberrant CpG island methylation along multistep gastric carcinogenesis. Lab Invest 2003:83:519-526.

7. Kim TY, Jong HS, Jung $Y$, et al. DNA hypermethylation in gastric cancer. Aliment Pharmacol Ther 2004;20(Suppl 1):131-142.

8. Kusano $M$, Toyota $M$, Suzuki $H$, et al. Genetic, epigenetic, and clinicopathologic features of gastric carcinomas with the $\mathrm{CpG}$ island methylator phenotype and an association with Epstein-Barr virus. Cancer 2006:106:1467-1479.

9. Kang SH, Bang YJ, Im YH, et al. Transcriptional repression of the transforming growth factor-beta type I receptor gene by DNA methylation results in the development of TGF-beta resistance in human gastric cancer. Oncogene 1999;18:7280-7286.

10. Song SH, Jong HS, Choi HH, et al. Methylation of specific $\mathrm{CpG}$ sites in the promoter region could significantly downregulate p16(INK4a) expression in gastric adenocarcinoma. Int J Cancer 2000;87:236-240.

11. Song SH, Jong HS, Choi HH, et al. Transcriptional silencing of cyclooxygenase-2 by hyper-methylation of the $5^{\prime} \mathrm{CpG}$ island in human gastric carcinoma cells. Cancer Res 2001;61:4628-4635.

12. Kim TY, Jong HS, Song SH, et al. Transcriptional silencing of the DLC-1 tumor suppressor gene by epigenetic mechanism in gastric cancer cells. Oncogene 2003;22:3943-3951.

13. Kim TY, Lee HJ, Hwang KS, et al. Methylation of RUNX3 in various types of human cancers and premalignant stages of gastric carcinoma. Lab Invest 2004;84:479-484.

14. Choi MC, Jong HS, Kim TY, et al. AKAP12/Gravin is inactivated by epigenetic mechanism in human gastric carcinoma and shows growth suppressor activity. Oncogene 2004;23:7095-7103.

15. Park J, Song SH, Kim TY, et al. Aberrant methylation of integrin alpha4 gene in human gastric cancer cells. Oncogene 2004;23:3474-3480.

16. Kim TY, Zhong $S$, Fields $C R$, et al. Epigenomic profiling reveals novel and frequent targets of aberrant DNA methylation-mediated silencing in malignant glioma. Cancer Res 2006;66:7490-7501.

17. Park YJ, Luger K. Structure and function of nucleosome assembly proteins. Biochem Cell Biol 2006;84:549-558.

18. Puffenberger EG, Hu-Lince D, Parod JM, et al. Mapping of sudden infant death with dysgenesis of the testes syndrome (SIDDT) by a SNP genome scan and identification of TSPYL loss of function. Proc Natl Acad Sci USA 2004;101:11689-11694.

19. Fujii-Nakata $T$, Ishimi $Y$, Okuda $A$, et al. Functional analysis of nucleosome assembly protein, NAP-1. The negatively charged
$\mathrm{COOH}$-terminal region is not necessary for the intrinsic assembly activity. J Biol Chem 1992;267:20980-20986.

20. Vogel T, Dittrich O, Mehraein Y, et al. Murine and human TSPYL genes: novel members of the TSPY-SET-NAP1L1 family. Cytogenet Cell Genet 1998;81:265-270.

21. Schnieders F, Dork T, Arnemann J, et al. Testis-specific protein, Y-encoded (TSPY) expression in testicular tissues. Hum Mol Genet 1996;5:1801-1807.

22. Ishimi $Y$, Kikuchi A. Identification and molecular cloning of yeast homolog of nucleosome assembly protein I which facilitates nucleosome assembly in vitro. J Biol Chem 1991;266:7025-7029.

23. Ishimi $Y$, Kojima $M$, Yamada $M$, et al. Binding mode of nucleosome-assembly protein (AP-I) and histones. Eur J Biochem 1987; 162:19-24.

24. Park YJ, Chodaparambil JV, Bao Y, et al. Nucleosome assembly protein 1 exchanges histone $\mathrm{H} 2 \mathrm{~A}-\mathrm{H} 2 \mathrm{~B}$ dimers and assists nucleosome sliding. J Biol Chem 2005;280:1817-1825.

25. Park YJ, Luger K. The structure of nucleosome assembly protein 1. Proc Natl Acad Sci USA 2006;103:1248-1253.

26. von Lindern $M$, van Baal $S$, Wiegant J, et al. Can, a putative oncogene associated with myeloid leukemogenesis, may be activated by fusion of its $3^{\prime}$ half to different genes: characterization of the set gene. Mol Cell Biol 1992;12:3346-3355.

27. Miyaji-Yamaguchi $M$, Kato $K$, Nakano $R$, et al. Involvement of nucleocytoplasmic shuttling of yeast Nap1 in mitotic progression. Mol Cell Biol 2003;23:6672-6684.

28. Oram SW, Liu XX, Lee TL, et al. TSPY potentiates cell proliferation and tumorigenesis by promoting cell cycle progression in HeLa and NIH3T3 cells. BMC Cancer 2006;6:154.

29. Chai Z, Sarcevic B, Mawson A, et al. SET-related cell division autoantigen-1 (CDA1) arrests cell growth. J Biol Chem 2001;276:33665-33674.

30. Wang GS, Hong CJ, Yen TY, et al. Transcriptional modification by a CASK-interacting nucleosome assembly protein. Neuron 2004;42:113-128.

31. Ozbun LL, You L, Kiang S, et al. Identification of differentially expressed nucleolar TGF-beta1 target (DENTT) in human lung cancer cells that is a new member of the TSPY/SET/NAP-1 superfamily. Genomics 2001;73:179-193.

32. Gallagher $\mathrm{WM}$, Bergin $\mathrm{OE}$, Rafferty $M$, et al. Multiple markers for melanoma progression regulated by DNA methylation: insights from transcriptomic studies. Carcinogenesis 2005;26:1856-1867.

33. Ku JL, Park JG. Biology of SNU cell lines. Cancer Res Treat 2005;37: 1-19.

34. Herman JG, Graff JR, Myohanen S, et al. Methylation-specific PCR: a novel PCR assay for methylation status of $\mathrm{CpG}$ islands. Proc Natl Acad Sci USA 1996;93:9821-9826.

35. Li LC, Dahiya R. MethPrimer: designing primers for methylation PCRs. Bioinformatics 2002;18:1427-1431.

36. Momparler RL. Cancer epigenetics. Oncogene 2003;22:6479-6483. 